\title{
Study of Coronary Sinus Flow Reserve Through Transesophageal Doppler Echocardiography in Normal Subjects
}

\author{
José Ramos Filho, José A. F. Ramires, Marko Turina, Caio J. Medeiros, Mário Lachat, Jeane Tsutsui
}

São Paulo, SP - Zurique, Switzerland

\begin{abstract}
Objective - To evaluate the Coronary Flow Reserve in the Coronary Sinus through transesophageal Doppler echocardiography in normal subjects.
\end{abstract}

Methods - We obtained technically adequate flow samples for analysis in 10 healthy volunteers ( $37 \pm 8$ years, 5 men) with no history of heart or systemic disease and with mean left ventricular mass index by transthoracic echocardiography of $87 \pm 18 \mathrm{~g} / \mathrm{m}^{2}$. Coronary sinus flow velocity was recorded within the coronary sinus with the patient in a resting condition and during intravenous adenosine infusion at a dose of $140 \mu \mathrm{g} / \mathrm{kg} / \mathrm{min}$ for 4 minutes. Recording of coronary sinus blood flow was possible in all cases with measurement of peak systolic, diastolic, and retrograde velocities (PSV, PDV, and PRV, $\mathrm{cm} / \mathrm{sec}$ ), mean systolic and diastolic velocities (MSV and $M D V, \mathrm{~cm} / \mathrm{sec})$, and systolic and diastolic velocity time integral (VTIS and VTID, $\mathrm{cm} / \mathrm{sec}$ ).

Results - The coronary flow reserve was calculated as the ratio between the blood flow in the basal state and the maximum measured hyperemic blood flow with adenosine infusion. Results are shown as mean and standard deviations. $(C F R=P S V+P D V-P R V /$ basal PSV $): 1^{\text {st }} \min =2.2 \pm 0.21 ; 2^{\text {nd }}$ $\min =3 \pm 0.3 ; 3^{\text {rd }} \min =3.4 \pm 0.37 ; 4^{\text {th }}$ min $=3.6 \pm 0.33$.

Conclusion - Although coronary sinus flow had significantly increased in the first minute, higher velocities were seen at third and fourth minutes, indicating that these should be the best times to study coronary sinus flow with intravenous adenosine in continuous infusion.

Keywords: transesophageal Doppler echocardiography, coronary sinus, coronary reserve

Heart Institute (InCor) University of São Paulo (Brazil) and Klinik für Herz - und Gefässchirurgie - Universitätsspital Zürich (Switzerland)

Mailing address: José Ramos Filho - Rua José Domingues, 420 - 12900-000 Bragança Paulista, SP - Brazil - E-mail: jramos-uti@uol.com.br
The capacity to increase coronary blood flow in response to an appropriate stimulus was determined by coronary flow reserve (CFR) or vasodilatating coronary reserve ${ }^{1}$. The most important methods for studying CFR are expansive and invasive, requiring time and appropriate laboratory equipment ${ }^{2-4}$. The main techniques used are flow measurement with intracoronary Doppler, positron emission tomography, and measuring coronary sinus flow by dilution term ${ }^{5}$. In the last few years, several authors have shown the possibility of velocity monitoring of coronary sinus flow (CSF) and CFR by transesophageal 2-dimensional Doppler echocardiography ${ }^{6,7}$.

Transesophageal Doppler echocardiography (TEE) is a semi-invasive procedure, with a low rate of complications, which evaluates CSF in real time with good reproducibility, and allows the monitoring of flow before and after the administration of a vasodilator $\mathrm{drug}^{8}$, providing important information about $\mathrm{CFR}^{9}$.

CSF by TEE can be monitored within a distance of 1 to $1.5 \mathrm{~cm}$, from the ostium with adequate alignment between the ultrasound beam and the coronary sinus (CS), where achieving adequate flow would be possible in more than $90 \%$ of cases ${ }^{6}$.

This method is useful for evaluating coronary flow (CF) in cases of specific lesion in the left anterior descending coronary artery ${ }^{10}$ or in situations with alterations in coronary circulation, as well as micro-circulation ${ }^{9,10}$, being of great applicability in evaluating patients with myocardial ischemia ${ }^{11}$. As far as drugs used to evaluate coronary vasodilator capacity, adenosine and dipyridamole are Known to be effective for intravenous application. Adenosine induces quick and massive vasodilatation mainly mediated by A1 and A2 purinergic receptors, previously called $P 1$ purinergic receptor subtype ${ }^{12,13}$, and may provoke depression of the sinoatrial and atrioventricular nodes, being dose-dependent, and diminution of arterial blood pressure ${ }^{14,15}$. Dipyridamole and adenosine have been used to evaluate CFR; both drugs produce comparable increases in the total and 
regional coronary flow. However, adenosine has fewer deleterious effects on cardiac electrophysiological properties ${ }^{16}$. Adenosine does not produce significant changes in ST segment, QT interval, myocardial creatine phosphate level or lactate level ${ }^{17}$. Adenosine has a short half-life and can be used in bolus or in continuous infusion with few side effects ${ }^{12,17}$. The effect of dipyridamole is slower and indirect, causing an increase in endogenous adenosine levels ${ }^{18}$. Recently, Kozàkovà et a $l^{19}$ observed a more prominent vasodilator response with continuous adenosine infusion, while the response to dipyridamole is dose-dependent, which may lead to an underestimation of the vasodilator response in patients with obstructive disease of the coronary sinus ${ }^{14}$.

The present study proposes an evaluation of the coronary blood flow reserve by transesophageal Doppler echocardiography examining coronary sinus flow velocity in the basal state and after intravenous adenosine in continuous infusion.

\section{Methods}

In the period from February through August 1997, we obtained technically adequate transesophageal Doppler flow samples for analysis of coronary sinus flow reserve in 10 healthy volunteers (mean age $37 \pm 8$ years, 5 men) with no history of heart or systemic disease and with mean left ventricular mass index analyzed by transthoracic echocardiography of $87 \mathrm{~g} / \mathrm{m}^{2} \pm 18$.

Coronary stenosis was supposedly excluded after a non-invasive investigation (clinical history, blood samples, basal electrocardiogram, normal treadmill test, normal effort thallium stress test, transthoracic and transesophageal Doppler echocardiography).

All subjects were requested to abstain from xanthinecontaining, food and drinks for at least 30 hours before the examination.

This study was approved by the Scientific Commission and Ethics Committee of the Heart Institute of the University of São Paulo (USP, Brazil) and the University of Zürich (Switzerland). Subjects were included after giving informed consent.

The control group was screened at the intensive care unit. During the study, a Dixtal monitor (Dixtal Biomedical Ind. \& co. Ltd., São Paulo, Brazil) continuously monitored blood pressure by non-invasive means with pulse oximetry and 3-lead electrocardiography. A 12-lead electrocardiogram was recorded in the basal state and at the end of every minute of vasodilator drug infusion. Injectable adenosine was provided by a laboratory ( Wyeth Lab., Paris, France) and infused with an OT-601 Pump (JMS Co., Ltd. Hiroshima, Japan) in a continuous solution for 4 minutes while the examination was recorded on videocassette tapes (Panasonic VCR) for later analysis.

A 3-liter/minute nasal catheter with oxygen and peripheral intravenous punching with a number $18 \mathrm{G}$ intravascular catheter was introduced into the right antecubital vein for administration of a vasodilator drug by continuous infusion. Blood pressure was assessed in the contralateral arm to that receiving adenosine at the end of every minute.

A transthoracic Doppler echocardiography with a 2.5 $\mathrm{MHz}$ transducer was performed before the semi-invasive procedure in all subjects.

After application of topical anesthesia of the oropharynx with xylocaine spray, the subject was placed on the left side, lightly sedated with intravenous midazolan in a dose of 0.15 to $0.20 \mathrm{mg} / \mathrm{kg}$. The transducer was lubricated with lidocaine gel and with flexing of the subject's head, a transesophageal $5 \mathrm{MHz}$ monoplane or biplane probe ATL 6000 and 4 Plus ( Ultramark ${ }^{\circledR} 6$ and 4 plus; Advanced Technology Laboratories, Inc.; Bothell, WA, U.S.A.) was introduced into the upper esophagus. The Doppler flow samples in the coronary sinus were obtained through the transversal and longitudinal plan approximately 30 to $40 \mathrm{~cm}$ from the incisors, with a light retroflexion of the transducer (fig. 1).

Investigation of flow velocity in the coronary sinus The pulsed Doppler sample was positioned in the coronary sinus within a distance of 10 to $15 \mathrm{~mm}$ from the ostium and at an angle between the Doppler beam and the long spindle of the coronary sinus of less than 30 degrees. Flow velocity was recorded during 06 consecutive cardiac cycles in prolonged expiration in the basal state and during adenosine infusion. The diameter of the coronary sinus was measured in the basal state and after administration of the vasodilator drug. Intravenous adenosine was infused at a dose of 140 $\mu \mathrm{g} / \mathrm{kg} / \mathrm{min}$, for 4 minutes, with recording of the flow velocity at the end of every minute. Anterograde systolic maximum velocity, anterograde diastolic maximum velocity, retrograde maximum velocity, systolic and diastolic velocity time integral (VTIS and VTID, cm/sec) were analyzed. The coronary flow reserve was calculated as the relation between coronary sinus flow in the basal state and in a state of maximum vasodilatation $(\mathrm{CFR}=\mathrm{PSV}+\mathrm{PDV}-\mathrm{PRV} / \mathrm{basal} \mathrm{PSV})$.

Primarily, all the variables were analyzed descriptively. For the continuos variables, this analysis was made through observation of minimum and maximum values, and

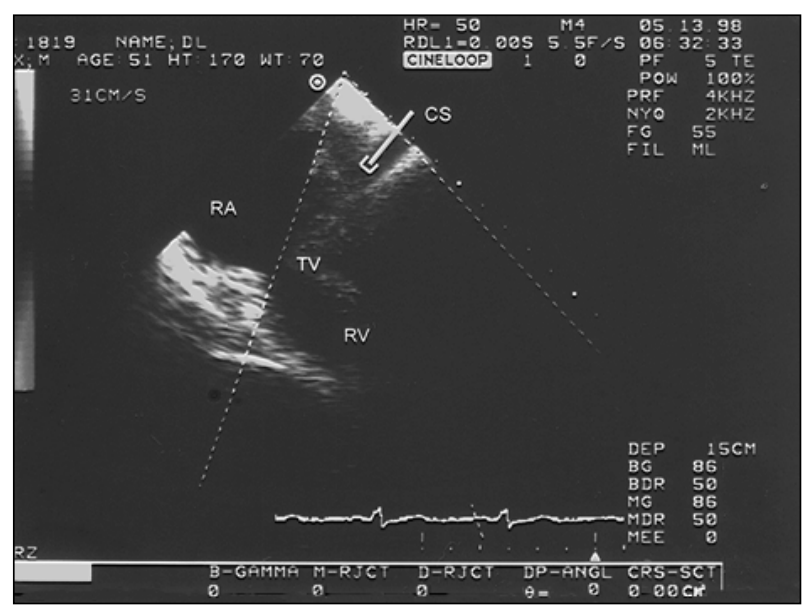

Fig. 1 - "Zoomed" 2-D image of the coronary sinus, from which the samples of flow were obtained. CS- coronary sinus; RA-right atrium; TV-tricuspid valve; RV-right ventricle. 
through calculation of averages and standard deviation. For the classificatory variables, the absolute and relative frequencies were calculated.

To check the group behavior regarding the conditions studied, the Profile Analysis Technique ${ }^{20}$ was used, which consists of the adjustment of a multi-varied linear model, by which the following hypotheses were tested: H01: The average response profile is parallel to the abscissa axis, ie, the evaluation condition factor has no effect. When H01 was rejected, the interest contrasts were tested.

In testing the above hypotheses, the Wilks' test was used, with approximation of the F statistic.

The comparisons between the averages of flow velocities in the control group were made through the nonpaired Student $t$ - test ${ }^{21}$ and the level used was $\mathrm{p}<0.001$. The significance level used for the tests was $5 \%$.

\section{Results}

The semi-invasive interventions occurred with no incidents in any subjects. Their recovery evolved in a stable manner without any clinical occurrences. No significant changes in left ventricular end-diastolic and systolic diameter and ejection fraction were encountered with adenosine.

During adenosine infusion we did not observe any side effects in the control group. In the beginning of the examination, a light increase occurred in heart rate and blood pressure. Two minutes later, blood pressure and heart rate were back to normal. At that moment, we began the adenosine infusion.

Adequate samples of flow velocity in the CS were obtained in 10 subjects of the control group (100\%) (fig. 2).

From the morphological point of view, 2 distinct Doppler sample curves in the CS were obtained, a biphasic curve with systolic predominant component that occurred in 8 out of 10 subjects $(80 \%)$ and a diastolic predominant component in 2 remaining subjects $(20 \%)$.

Through quantitative analyses, flow samples in the control group were investigated as were the peak systolic

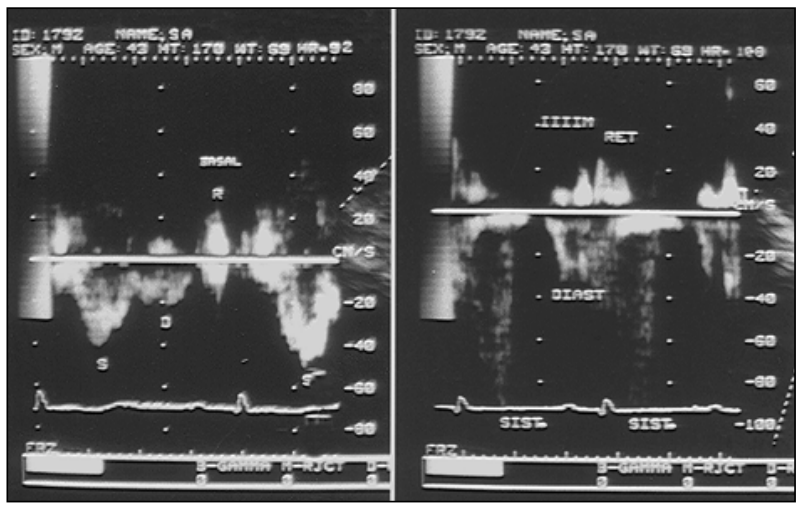

Fig. 2 - Normal biphasic Doppler flow pattern recording in coronary sinus characterized by systolic and diastolic anterograde flow as well as a short period of end-diastolic retrograde flow. Coronary sinus flow in the basal state and at third minute during adenosine infusion. S- systolic flow; D- diastolic flow; Rretrograde flow.

velocity, peak diastolic velocity, mean systolic velocity, mean diastolic velocity, and systolic and diastolic velocity time-integral (VTIS and VTID. cm/s). A short diastolic retrograde peak flow was observed in the CS in all subjects. The coronary flow velocity, variables (PDV, MDV, VTID, PSV, MSV, VTI S) and coronary flow reserve, increased in all subjects during adenosine infusion (tab. I).

Analyses of heart rate and blood pressure during adenosine infusion - We believe that no interference with heart rate and mean blood pressure occurred in CSFR; however, a light increase in the heart rate and a decrease in blood pressure were observed during adenosine infusion.

Coronary flow reserve in normal subjects - Coronary sinus flow reserve had significantly increased during the first minutes. Higher velocities were seen at the third and fourth minutes, indicating that these should be the best times to study coronary sinus flow with intravenous adenosine during continuous infusion.

Results are shown as mean and standard deviations $\left(2.2 \pm 0.211^{\circ} \mathrm{min}, 3.0 \pm 0.302^{\circ} \mathrm{min}, 3.4 \pm 0.373^{\circ} \mathrm{min}, 3.6 \pm 0.334^{\circ}\right.$ min)(fig. 3).

\begin{tabular}{|c|c|c|c|c|c|c|c|c|c|c|}
\hline \multicolumn{11}{|c|}{ Results are shows as mean and standard deviations } \\
\hline & PSV & PDV & PRV & MSV & MDV & VTIS & VTID & HR & SBP & DBP \\
\hline \multirow[t]{2}{*}{ Rest } & 39.3 & 25.3 & 23.2 & 33.3 & 21.3 & 7.2 & 4.3 & 77 & 123 & 78 \\
\hline & \pm 7.8 & \pm 3.6 & \pm 4.8 & \pm 5.9 & \pm 3.9 & \pm 1.6 & \pm 1.4 & \pm 14 & \pm 12 & \pm 17 \\
\hline Aden & 68.6 & 46.7 & 25.7 & 53.5 & 37.3 & 14.9 & 9.5 & 82 & 115 & 76 \\
\hline $1 \mathrm{~min}$ & \pm 18.4 & \pm 17.3 & \pm 4.1 & \pm 15.2 & \pm 18.5 & \pm 6.7 & \pm 2.8 & \pm 23 & \pm 16 & \pm 15 \\
\hline Aden & 88.7 & 58.7 & 28.4 & 66.3 & 44.9 & 16.8 & 13.8 & 95 & 115 & 72 \\
\hline $2 \mathrm{~min}$ & \pm 20.4 & \pm 8.9 & \pm 4.3 & \pm 21.4 & \pm 11.3 & \pm 7.2 & \pm 6.5 & \pm 16 & \pm 24 & \pm 17 \\
\hline Aden & 106.8 & 59.8 & 32 & 73.7 & 45.8 & 21.7 & 14.4 & 94 & 112 & 73 \\
\hline $3 \mathrm{~min}$ & \pm 22.3 & \pm 15.4 & \pm 7.5 & \pm 24.3 & \pm 12.1 & \pm 8.1 & \pm 7.3 & \pm 16 & \pm 16 & \pm 19 \\
\hline Aden & 111.4 & 66.45 & 35.4 & 82.8 & 52.1 & 23.8 & 15.6 & 90 & 110 & 70 \\
\hline $4 \mathrm{~min}$ & \pm 19.2 & \pm 19.3 & \pm 8.2 & \pm 13.2 & \pm 15.3 & \pm 6.4 & \pm 6.4 & \pm 17 & \pm 16 & \pm 11 \\
\hline
\end{tabular}




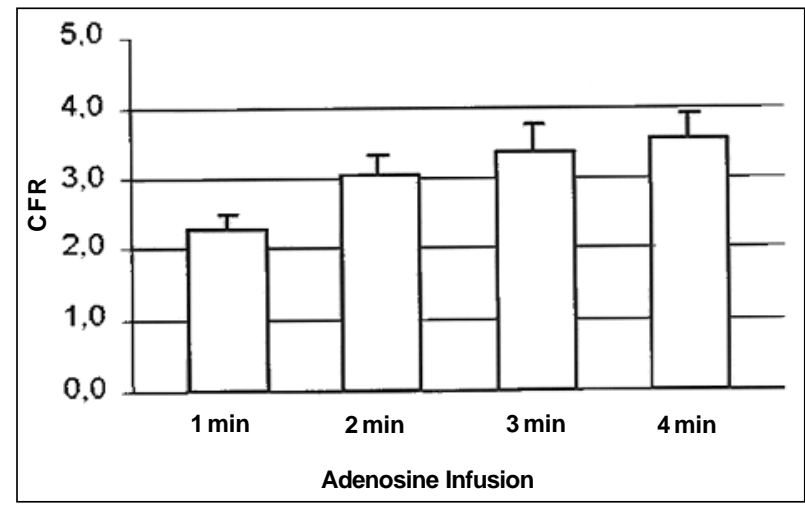

Fig. 3 - Coronary flow reserve (CFR) in coronary sinus in normal subjects.

\section{Discussion}

In the last 2 decades, a surprising development has taken place in transesophageal Doppler echocardiography (TEE), making it possible to measure blood flow velocity (BFV) in the coronary arteries and coronary sinus. In addition, the infusion of intravenous vasodilator drugs is now possible during the examination, making it possible to increase the $\mathrm{BFV}$, allowing comparisons between flows during the basal state and flow during maximum dilatation.

TEE with adenosine infusion constitutes a new semi-invasive method that permits the determination of CFR through blood flow samples in normal subjects and in a variety of cardiac diseases ${ }^{6,19,22}$ making the comparis with other methods already used as described in the introduction ${ }^{4,23}$.

Determination of CFR is an established method for assessing information about coronary artery stenosis ${ }^{24}$ and has been used to evaluate normal subjects and results of PTCA $^{25}$ and, we propose through this investigation, to evaluate healthy volunteers to compare subsequently with results obtained in patients after PTCA and bypass surgery.

To evaluate CFR, we chose adenosine because it is an effective drug with few side effects when compared with other drugs. In 1991, Illiceto et al ${ }^{8}$ described for the first time the calculation of CFR by TEE recordings of CFV in the LAD in patients without and in patients with coronary artery disease. The CFR in the control group without coronary disease was $3.2 \pm 1.0$, and in patients with significant stenosis of the LAD it was $1.5 \pm 1.5$.

In the same year, Kern et al ${ }^{17}$ investigated CFR with adenosine infusion in a dose of 50, 100 and $150 \mu \mathrm{g} / \mathrm{kg}$ and measured CFV in the basal state and the hyperemic response for 3 minutes in patients without coronary disease and observed an increase in the flow velocity with the maximal dose infused and CFR was $2.94 \pm 1.5$.

In 1995, Redberg et al ${ }^{26}$ reported the possibility of flow monitoring in the LAD in approximately $70 \%$ to $80 \%$ of cases. Tsutsui et al ${ }^{27}$ demonstrated CFR during adenosine infusion in the LAD in a control group of healthy volunteers and observed similar results - to those of previous studies. When CFR is $>2$, no critical LAD stenosis exists ${ }^{28}$.
Doppler flow sample measurements in the CS during intravenous dipyridamole were obtained for assessment of CSF reserve comparing patients without cardiac disease and patients with idiopathic dilated cardiomyopathy ${ }^{6}$.

In Siostrzonek et al ${ }^{6}$ control group, 9 patients with suspected cerebral embolism but without cardiac disease had a CFR of $3.4 \pm 2$, and a CFR value of 1.8 was the reference used to compare patients without cardiac disease and patients with dilated cardiomyopathy. In that study, a significant increase in CSF $>95 \%$ was observed in the control group as was an increase in CSF and CFR only in 3 of 9 patients with dilated cardiomyopathy.

In 1997, Mundigler et al ${ }^{7}$ evaluated the CFR in 2 different groups of patients. Fourteen neurosurgical patients without cardiac disease composed their control group, and 2 distinct samples were considered for their studies in CS. When CFR was calculated through anterograde flow velocity-time integral, the value was $2.59 \pm 0.74$, and when calculated through anterograde maximum flow velocity, the CFR was $1.76 \pm 0.52$. In some studies ${ }^{6,7,22}$ the $C F R$ values by TEE in the coronary sinus were significantly reduced in patients with idiopathic dilated cardiomyopathy, syndrome $\mathrm{X}$, and significant LAD stenosis when compared with other groups without cardiac disease.

In the present study, Doppler flow samples monitoring in real time beat-to-beat in the CS were feasible in all subjects, and CFR was calculated as the ratio between blood flow in the basal state and the maximum measured hyperemic blood flow with adenosine infusion.

Retrograde peak flow was present in all subjects and was included in the calculation of CFR in the CS (CFR $=$ PSV + PDV -PRV/PSV). The evaluation of flow velocity and CFR in our control group was superior to the ones described so far because our control group was formed of young healthy volunteers without neurological and cardiac disease, and from the first minutes of adenosine infusion, we observed CFR $>2$. May be that is the reason why we found CFR more homogenous during the entire study protocol.

Our original study shows the potential reliability of the TEE method before and after intravenous adenosine infusion in normal subjects with supposedly normal left ventricular function. The measurement technique of CFR in the coronary sinus of the present study is the first one described. Technically, the achievement of samples in the coronary sinus was easier to obtain in spite of some interference with the Doppler signal.

Despite the fact that the TEE method had been studied by several authors who demonstrated its efficiency, limitations do exist: 1) Our control group was formed by young, healthy volunteers, and we evaluated a small group; however, the analyzed group had uniform pattern flow samples; 2) The coronary sinus flow measurement was sometimes difficult due to the heart and breathing movements of the subjects mainly because of intolerance to the examination. 3) Subjects, who are lightly sedated, sometimes could not cooperate in an adequate way during the examination; 4) In several homogenous cardiac cycles, CSFV had varia- 
tions dependent on the breathing cycles, which modified the position of the transducer and changed the patterns of the flow samples.

Therefore, it is important to consider the position of the transducer to obtain samples as close as possible to the expected average.

New data about the subjects condition will be possible through subsequent studies, suggesting a routine application of the method due to low risk and low cost, and be- cause TEE with adenosine infusion is rapid, safe, and effective.

Conclusion - In our Doppler echocardiography study we demonstrate that coronary sinus flow velocity and coronary flow reserve increased during intravenous adenosine infusion in the coronary sinus in normal subjects and that the systolic variables (PSV, MSV and VTI S) were superior to the diastolic (PDV, MVD and VTID) ones.

\section{References}

1. Gould KL, Lipscomb K, Hamilton GW. Physiologic basis for assessing critical coronary stenosis. Instantaneous flow response and regional distribution during coronary hyperemia as measures of coronary flow reserve. Am J Cardiol 1974; 33: 87-94

2. Ganz W, Tamura K, Marcus HS, Donoso R, Yoshida S, Swan HJC. Measurement of coronary sinus blood flow velocity and vasodilator reserve in man. Circulation 1971; 44: 181-95.

3. Wilson RF, Laughlin DE, Ackell PH, et al. Transluminal, subselective measurement of coronary artery blood flow velocity and vasodilator reserve in man. Circulation 1985; 72: 82-92.

4. White CM, Wilson RF, Marcus ML. Methods of measuring myocardial blood flow in humans. Prog Cardivasc Dis 1988; 31: 79-94.

5. Bergmann JR, Fox RA, Rand AL, et al. Qualification of regional myocardial blood flow in vivo with oxygen-15 and positron emission tomography. Circulation 1984; 70: 724-33.

6. Siostrzonek P, Kranz A, Heinz G, et al. Noninvasive estimation of coronary flow reserve by transesophageal Doppler measurement of coronary sinus flow. Am J Cardiol 1993; 72: 1334-36.

7. Mundigler G, Zehetgruber M, Christ G, Siostrzonek P. Comparison of transesophageal coronary sinus and left anterior descending coronary artery Doppler measurements for the assessment of coronary flow reserve. Clin Cardiol 1997;20: 225-31.

8. Illiceto S, Marangelli V, Memmola C, Rizzon P. Transesophageal Doppler echocardiography evaluation of coronary blood velocity in baseline conditions and during dipyridamole induced coronary vasodilatation. Circulation 1991; 83: 61-9.

9. Palombo C, Kozàkovà M, Vassale C, Distante A, L'Abbate. Coronary pressureflow diagrams during maximal vasodilatation as assessed by transesophageal Doppler of left coronary artery. A new tool for evolution of absolute coronary flow reserve. J Am Coll Cardiol 1994; 115.

10. Hutchinson SJ, Marwah OS, Posly KM, Kawanishi DT, Chandraratna PAN. Coronary flow velocity reserve after sucessful ballon angioplasty and directional atherectomy. Echocardiography 1995; 12: 591-603.

11. Ramos JF, Turina M, Ramires JA, et al. Demonstration of Coronary Flow Reserve Before and After Angioplasty and Bypass Surgery by Transesophageal Doppler Echocardiography Method. Inaugural-Dissertation Universitätsspital Zürich, 1999; 17: 42-45.

12. Wilson RF, Wyche K, Christensen BV,Zimmer S, Laxson DD. Effects of adenosine on human coronary arterial circulation. Circulation 1990; 82: 1595-606.

13. Hori M, Kitakaze M. Adenosine, the heart and coronary circulation. Hypertension 1991; 18: 565-74.

14. Rosen JD, Quillen JE, Lopez AG, Stenberg RG, Talman CL, Winniford MD. Comparison of coronary vasodilatation with intravenous dipyridamole and adenosine. J Am Coll Cardiol 1991; 18: 485-91.
15. Abreu A, Hahmarian JJ, Nishimura S, Bryce TM, Verani MS. Tolerance and safety of pharmacologic coronary vasodilation whith adenosine in association with thallium-201 scintigraphy in patients with suspected coronary artery disease. $\mathrm{J}$ Am Coll Cardiol 1991: 730-5.

16. Chistensen CW, Rosen LB, Gal RA, Haseeb M, Lassar TA, Port SC. Coronary vasodilator reserve. Comparison of the effect of papaverine and adenosine on coronary flow, ventricular function and myocardial metabolism. Circulation 1991; 83: 294-303.

17. Kern MJ, Deligonul U, Tatineni S, Serota H, Aguirre F, Hilton TC. Intravenous adenosine: continuous infusion and low dose bolus administration for determination of coronary vasodilator reserve in patients with and without coronary artery disease. J Am Coll Cardiol 1991; 18: 718-29.

18. Knabb RM, Gidday JM, Ely SW, Rubio R, Berne RM. Effects of dipyridamole on myocardial adenosine and active hyperemia. Am J Physiol 1984; 247: H804-10.

19. Kozàkovà $\mathrm{M}$, Palombo $\mathrm{C}$, Pratali L, et al. Assessment of coronary reserve by Transesophageal Doppler echocardiography. Direct comparsion between different modalities of dipyridamole and adenosine administration. Eur Heart J 1997; 18: 514-23.

20. Timm NH, Multivariate Analysis with Applications in Education and Psychology. Monterrey, CA Brooks/ Cole, 1975.

21. Rosner, B. Fundamentals of Biostatistics. $2^{\text {nd }}$ edition, Boston: PWS Publisher, 1986.

22. Zehetgruber M, Mundigler G, Chirst G, et al. Estimation of coronary flow reserve by transesophageal coronary sinus Doppler measurements in patients with syndrome $\mathrm{X}$ and patients with significant left coronary artery disease. J Am Coll Cardiol 1995; 25: 1039-45.

23. Sütsch G, Hess OM, Franzeck UK, et al. Cutaneous and coronary flow reserve in patients with microvascular angina, J Am Coll Cardiol 1992; 20: 78-84.

24. Marcus ML, Chilian WM, Kanatuka H, Dellsperger KC, et at - Understanding the coronary circulation through studies at the microvascular level. Circulation 1990; 82:1-7.

25. Paraskevaidis IA, Katritsis DG, Tsiapras DP, et al. Coronary flow reserve assessed by transesophageal echocardiography identifies early restenosis of the left anterior descending coronary artery angioplasty. Am J Cardiol 1997; 79:803-7.

26. Redberg RF, Sobol Y, Chou TM, et al. Adenosine - induced coronary vasodilatation during transesophageal Doppler echocardiography. Rapid and safe measurement of coronary flow reserve ratio can predict significant left anterior descending coronary stenosis. Circulation 1995; 92: 190-6.

27. Tsutsui JM, Helaehil SM, Correa MC, et al. Evaluation of coronary blood flow reserve by transesophageal Doppler echocardiography in normal subjects. Heart Institute University of São Paulo, Brazil 1997: 13.

28. Taams MA, Gussenhoven FJ, Cornel JH, et al. Detection of left coronary stenosis by transesophageal echocardiography. Eur Heart J 1988; 9: 1162-6. 